\title{
Erratum
}

\section{In: Acknowledgments}

World J. Surg. 17, 813, 1993

In the section listing the retiring Consultant Board Members, regretfully the name of Dr. W.H. Brummelkamp was omitted. 